Research Article

\title{
Fractional Ostrowski Type Inequalities via Generalized Mittag-Leffler Function
}

\author{
Xinghua You, ${ }^{1}$ Ghulam Farid $\left(D,{ }^{2}\right.$ and Kahkashan Maheen ${ }^{2}$ \\ ${ }^{1}$ Department of Mathematics and Physics, Nanjing Institute of Technology, Nanjing 211167, China \\ ${ }^{2}$ Department of Mathematics, COMSATS University Islamabad, Attock Campus, Islamabad, Pakistan
}

Correspondence should be addressed to Ghulam Farid; faridphdsms@hotmail.com

Received 23 April 2020; Revised 12 May 2020; Accepted 13 May 2020; Published 4 June 2020

Guest Editor: Praveen Agarwal

Copyright (c) 2020 Xinghua You et al. This is an open access article distributed under the Creative Commons Attribution License, which permits unrestricted use, distribution, and reproduction in any medium, provided the original work is properly cited.

If we study the theory of fractional differential equations then we notice the Mittag-Leffler function is very helpful in this theory. On the contrary, Ostrowski inequality is also very useful in numerical computations and error analysis of numerical quadrature rules. In this paper, Ostrowski inequalities with the help of generalized Mittag-Leffler function are established. In addition, bounds of fractional Hadamard inequalities are given as straightforward consequences of these inequalities.

\section{Introduction}

Exponential function plays a vital role in the theory of integer order differential equations. The symbol $E_{\alpha}(z)$ is well known as the Mittag-Leffler function and it is a generalization of exponential function. It occurs in the solutions of fractional differential equations such as exponential function which exists in the solutions of differential equations. Due to its importance, Mittag-Leffler function is generalized by many mathematicians: For example, Wiman [1], Prabhakar [2], Shukla and Prajapati [3], Salim [4], Salim and Faraj [5], and Rahman et al. [6]. Mittag-Leffler function is also used in the formation of fractional integral operators. These fractional integral operators provide generalizations of fractional differential equations and modeling of dynamic systems. Fractional integral operators also play a vital role in the advancement of classical mathematical inequalities. For example, Hadamard inequality, Ostrowski inequality, Gruss inequality, and many others have been presented for fractional integral and derivative operators, see [7-16]. The aim of this paper is to study well-known Ostrowski inequality for an integral operator which is directly associated with many fractional integral operators defined in near past.
Recently, in [7], Andrić et al. defined the extended generalized Mittag-Leffler function $E_{\mu, \sigma, l}^{\gamma, \delta, k, c}(. ; p)$ as follows.

Definition 1. Let $\mu, \alpha, l, \gamma, c \in \mathbb{C}, \mathfrak{R}(\mu), \mathfrak{R}(\alpha), \mathfrak{R}(l)>0$, and $\mathfrak{R}(c)>\mathfrak{R}(\gamma)>0$ with $p \geq 0, \delta>0$, and $0<k \leq \delta+\mathfrak{R}(\mu)$. Then, the extended generalized Mittag-Leffler function $E_{\mu, \alpha, l}^{\gamma, \delta, c}(t ; p)$ is defined as

$$
E_{\mu, \alpha, l}^{\gamma, \delta, c}(t ; p)=\sum_{n=0}^{\infty} \frac{\beta_{p}(\gamma+n k, c-\gamma)}{\beta(\gamma, c-\gamma)} \frac{(c)_{n k}}{\Gamma(\mu n+\alpha)} \frac{t^{n}}{(l)_{n \delta}},
$$

where $\beta_{p}$ is the generalized beta function defined as $\beta_{p}(x, y)=\int_{0}^{1} t^{x-1}(1-t)^{y-1} e^{-(p / t(1-t))} \mathrm{d} t$ and $(c)_{n k}$ is the Pochhammer symbol given by $(c)_{n k}=(\Gamma(c+n k)) / \Gamma(c)$.

The corresponding left- and right-sided generalized fractional integrals $\epsilon_{\mu, \alpha, l, \omega, a^{+}}^{\gamma, \delta, c, c}$ and $\epsilon_{\mu, \alpha, l, \omega, b^{-}}^{\gamma, \delta, k, c}$ are defined as follows.

Definition 2 (see [7]). Let $\omega, \mu, \alpha, l, \gamma, c \in \mathbb{C}, \mathfrak{R}(\mu), \mathfrak{R}(\alpha)$, $\mathfrak{R}(l)>0, \quad \mathfrak{R}(c)>\mathfrak{R}(\gamma)>0 \quad$ with $\quad p \geq 0, \quad \delta>0, \quad$ and $0<k \leq \delta+\Re(\mu)$. Let $\psi_{1} \in L_{1}[a, b]$ and $x \in[a, b]$. Then, the generalized fractional integrals $\epsilon_{\mu, \alpha, l, c, a^{+}}^{\gamma, \delta} \psi_{1}$ and $\epsilon_{\mu, \alpha, l, \omega, b^{-}}^{\gamma, \delta, k} \psi_{1}$ are defined as 
$\left(\epsilon_{\mu, \alpha, l, \omega, a^{+}}^{\gamma, \delta, k, c} \psi_{1}\right)(x ; p)=\int_{a}^{x}(x-t)^{\alpha-1} E_{\mu, \alpha, l}^{\gamma, \delta, k, c}\left(\omega(x-t)^{\mu} ; p\right) \psi_{1}(t) \mathrm{d} t$

$\left(\epsilon_{\mu, \alpha, l, \omega, b^{-}}^{\gamma, \delta, k, c} \psi_{1}\right)(x ; p)=\int_{x}^{b}(t-x)^{\alpha-1} E_{\mu, \alpha, l}^{\gamma, \delta, k, c}\left(\omega(t-x)^{\mu} ; p\right) \psi_{1}(t) \mathrm{d} t$.

Recently, Farid defined a unified integral operator in [17] (also see [18]). This unifies several kinds of fractional and conformable integrals in a compact formula and is given as follows.

Definition 3. Let $\psi_{1}, \psi_{2}:[a, b] \longrightarrow \mathbb{R}, 0<a<b$, be the functions such that $\psi_{1}$ be positive and $\psi_{1} \in L_{1}[a, b]$, and $\psi_{2}$ be differentiable and strictly increasing. Also, let $\phi / x$ be an increasing function on $[a, \infty)$ and $\alpha, l, \gamma, c \in \mathbb{C}$, $\mathfrak{R}(\alpha), \mathfrak{R}(l)>0, \quad \mathfrak{R}(c)>\mathfrak{R}(\gamma)>0, \quad p, \mu, \delta \geq 0, \quad$ and $0<k \leq \delta+\mu$. Then, for $x \in[a, b]$ the left and right integral operators are defined by

$$
\left({ }_{g} F_{\mu, \alpha, l, a^{+}}^{\phi, \gamma, \delta, c, c} \psi_{1}\right)(x, \omega ; p)=\int_{a}^{x} K_{x}^{y}\left(E_{\mu, \alpha, l}^{\gamma, \delta, k, c}, g ; \phi\right) \psi_{1}(y) d\left(\psi_{2}(y)\right),
$$

$$
\left({ }_{g} F_{\mu, \alpha, l, b^{-}}^{\phi, \gamma, \delta, k, c} \psi_{1}\right)(x, \omega ; p)=\int_{x}^{b} K_{y}^{x}\left(E_{\mu, \alpha, l}^{\gamma, \delta, k, c}, g ; \phi\right) \psi_{1}(y) d\left(\psi_{2}(y)\right)
$$

where $\quad K_{x}^{y}\left(E_{\mu, \alpha, l}^{\gamma, \delta, k, c}, g ; \phi\right)=\left(\left(\phi\left(\psi_{2}(x)-\psi_{2}(y)\right)\right) /\left(\psi_{2}(x)-\right.\right.$ $\left.\left.\psi_{2}(y)\right)\right) E_{\mu, \alpha, l}^{\gamma, \delta, c, c}\left(\omega\left(\psi_{2}(x)-\psi_{2}(y)\right)^{\mu} ; p\right)$.

The following definition can be deduced from Definition 3 (see [16]).

Definition 4. Let $\psi_{1}, \psi_{2}:[a, b] \longrightarrow \mathbb{R}, 0<a<b$, be the functions such that $\psi_{1}$ be positive and $\psi_{1} \in L_{1}[a, b]$, and $\psi_{2}$ be differentiable and strictly increasing and $\alpha, l, \gamma, c \in \mathbb{R}_{+}, c>\gamma, p, \mu, \delta \geq 0$ and $0<k \leq \delta+\mu$. Then, for $x \in[a, b]$ the left and right integral operators are defined by

$$
\begin{aligned}
& \left({ }_{\psi_{2}} \Upsilon_{\mu, \alpha, l, \omega, a^{+}}^{\gamma, \delta, k, c} \psi_{1}\right)(x ; p)=\int_{a}^{x}\left(\psi_{2}(x)-\psi_{2}(t)\right)^{\alpha-1} E_{\mu, \alpha, l}^{\gamma, \delta, k, c}\left(\omega\left(\psi_{2}(x)-\psi_{2}(t)\right)^{\mu} ; p\right) \times \psi_{1}(t) \psi_{2}^{\prime}(t) \mathrm{d} t, \\
& \left({ }_{\psi_{2}} \Upsilon_{\mu, \alpha, l, \omega, b^{-}}^{\gamma, \delta, k, c} \psi_{1}\right)(x ; p)=\int_{x}^{b}\left(\psi_{2}(t)-\psi_{2}(x)\right)^{\alpha-1} E_{\mu, \alpha, l}^{\gamma, \delta, k, c}\left(\omega\left(\psi_{2}(t)-\psi_{2}(x)\right)^{\mu} ; p\right) \times \psi_{1}(t) \psi_{2}^{\prime}(t) \mathrm{d} t .
\end{aligned}
$$

It can be noted that

$$
\begin{aligned}
& \left({ }_{\psi_{2}} \Upsilon_{\mu, \alpha, l, \omega, a^{+}}^{\gamma, \delta, k, c} 1\right)(x ; p)=\left(\psi_{2}(x)-\psi_{2}(t)\right)^{\alpha-1} E_{\mu, \alpha, l}^{\gamma, \delta, k, c}\left(\omega\left(\psi_{2}(x)-\psi_{2}(t)\right)^{\mu} ; p\right), \\
& \left({ }_{\psi_{2}} \Upsilon_{\mu, \alpha, l, \omega, b^{-}}^{\gamma, \delta, k, c} 1\right)(x ; p)=\left(\psi_{2}(t)-\psi_{2}(x)\right)^{\alpha-1} E_{\mu, \alpha, l}^{\gamma, \delta, k, c}\left(\omega\left(\psi_{2}(t)-\psi_{2}(x)\right)^{\mu} ; p\right) .
\end{aligned}
$$

In the following, we state the Ostrowski inequality which is proved by Ostrowski [19] in 1938.

Theorem 1. Let $\psi_{1}: I \longrightarrow \mathbb{R}$, where $I$ is an interval in $\mathbb{R}$, be a mapping differentiable in $I^{o}$, the interior of $I$ and $a, b \in I^{o}$, $a<b$. If $\left|\psi_{1}^{\prime}(t)\right| \leq M$ for all $t \in[a, b]$, then for $x \in[a, b]$ we have

$$
\left|\psi_{1}(x)-\frac{1}{b-a} \int_{a}^{b} \psi_{1}(t) \mathrm{d} t\right| \leq\left[\frac{1}{4}+\frac{(x-((a+b) / 2))^{2}}{(b-a)^{2}}\right](b-a) M .
$$

The Ostrowski inequality has been studied by many researchers to obtain its refinements, generalizations, and extensions. Also, their applications are analyzed for establishing the bounds of relations among special means and for estimations of numerical quadrature rules. For recent developments of Ostrowski inequality, we refer the reader to $[8,9,11,20-26]$ and references therein.

In Section 2, fractional version of Ostrowski inequalities with the help of Mittag-Leffler function has been established. The presented results may be useful in the study of fractional integral operators and their applications. Also, the error bounds of fractional Hadamard inequalities are presented in Section 3.

\section{Main Results}

First, we establish the following lemma for extended generalized Mittag-Leffler function.

Lemma 1. If $\omega, \mu, \alpha, l, \gamma, c \in C, \mathfrak{R}(\mu), \mathfrak{R}(\alpha), \mathfrak{R}(l)>0$, $\mathfrak{R}(c)>\mathfrak{R}(\gamma)>0$ with $p \geq 0, \delta>0$ and $0<k<\delta+\mathfrak{R}(\mu)$, then 


$$
\begin{gathered}
\left(\frac{d}{d t}\right)\left[\left(\psi_{2}(t)\right)^{\alpha-1} E_{\mu, \alpha, l}^{\gamma, \delta, k, c}\left(\omega \psi_{2}(t)^{\mu} ; p\right)\right] \\
=\psi_{2}^{\prime}(t) \psi_{2}(t)^{\alpha-2} E_{\mu, \alpha-1, l}^{\gamma, \delta, k, c}\left(\omega \psi_{2}(t)^{\mu} ; p\right) .
\end{gathered}
$$

Proof. We have

$$
\begin{aligned}
\left(\frac{\mathrm{d}}{\mathrm{d} t}\right)\left[\left(\psi_{2}(t)\right)^{\alpha-1} E_{\mu, \alpha, l}^{\gamma, \delta, c, c}\left(\omega \psi_{2}(t)^{\mu} ; p\right)\right] & =\sum_{n=0}^{\infty} \frac{\beta_{p}(\gamma+n k, c-\gamma)}{\beta(\gamma, c-\gamma)} \frac{(c)_{n k}}{\Gamma(\mu n+\alpha)} \frac{\omega^{n}(\mu n+\alpha-1)\left(\psi_{2}(t)\right)^{\mu n+\alpha-2} \psi_{2}^{\prime}(t)}{(l)_{n \delta}} \\
& =\sum_{n=0}^{\infty} \frac{\beta_{p}(\gamma+n k, c-\gamma)}{\beta(\gamma, c-\gamma)} \frac{(c)_{n k}}{\Gamma(\mu n+\alpha-1)} \frac{\omega^{n}\left(\psi_{2}(t)\right)^{\mu n+\alpha-2} \psi_{2}^{\prime}(t)}{(l)_{n \delta}}
\end{aligned}
$$

After simple computation, one can obtain (11).

Next, we give the generalized fractional Ostrowski type inequality containing extended generalized Mittag-Leffler function.

Theorem 2. Let $\psi_{1}: I \longrightarrow \mathbb{R}$, where $I$ is an interval in $\mathbb{R}$, be a mapping differentiable in $I^{o}$, the interior of $I$ and $a, b \in I^{o}$, $a<b$. If $\psi_{1}$ is an integrable function, $\left|\psi_{1}^{\prime}\left(\psi_{2}(t)\right)\right| \leq M$ for all $t \in[a, b]$ and $\psi_{2}:[a, b] \longrightarrow \mathbb{R}$ be an increasing and positive function on $(a, b]$, having continuous derivative $\psi_{2}^{\prime}$ on $(a, b)$, then for $\alpha, \beta \geq 1$, the following inequality for fractional integrals (6) and (7) holds:

$$
\begin{aligned}
& \mid \psi_{1}\left(\psi_{2}(x)\right)\left(\left(\psi_{2}(b)-\psi_{2}(x)\right)^{\beta-1} E_{\mu, \beta, l}^{\gamma, \delta, k, c}\left(\omega\left(\psi_{2}(b)-\psi_{2}(x)\right)^{\mu} ; p\right)+\left(\psi_{2}(x)-\psi_{2}(a)\right)^{\alpha-1}\right. \\
& \quad \times E_{\mu, \alpha, l}^{\gamma, \delta, k, c}\left(\omega\left(\psi_{2}(x)-\psi_{2}(a)\right)^{\mu} ; p\right)-\left(\left({ }_{\psi_{2}} Y_{\mu, \alpha-1, l, \omega, a^{+}}^{\gamma, \delta, k, c} \psi_{1} \circ \psi_{2}\right)(x ; p)+\left({ }_{\psi_{2}} Y_{\mu, \beta-1, l, \omega, b^{-}}^{\gamma, \delta, k, c} \psi_{1} \circ \psi_{2}\right)(x ; p)\right) \mid \\
& \quad \leq M\left(\left(\psi_{2}(x)-\psi_{2}(a)\right)^{\alpha} \times E_{\mu, \alpha, l}^{\gamma, \delta, c, c}\left(\omega\left(\psi_{2}(x)-\psi_{2}(a)\right)^{\mu} ; p\right)+\left(\psi_{2}(b)-\psi_{2}(x)\right)^{\beta} E_{\mu, \beta, l}^{\gamma, \delta, k, c}\left(\omega\left(\psi_{2}(b)-\psi_{2}(x)\right)^{\mu} ; p\right)\right. \\
& \left.\quad-\left(\left(\psi_{2} \Upsilon_{\mu, \alpha-1, l, \omega, a^{+}}^{\gamma, \delta, k, c}\right)(x ; p)+{ }_{\psi_{2}} \Upsilon_{\mu, \beta-1, l, \omega, b^{-}}^{\gamma, \delta, k, c}\right)(x ; p)\right) .
\end{aligned}
$$

Proof. Let $x \in[a, b], t \in[a, x]$, and $\alpha \geq 1$. Then, the following inequality holds for the monotonically increasing function $\psi_{2}$ and the Mittag-Leffler function (1):

$$
\left(\psi_{2}(x)-\psi_{2}(t)\right)^{\alpha-1} E_{\mu, \alpha, l}^{\gamma, \delta, k, c}\left(\omega\left(\psi_{2}(x)-\psi_{2}(t)\right)^{\mu} ; p\right) \psi_{2}^{\prime}(t) \leq\left(\psi_{2}(x)-\psi_{2}(a)\right)^{\alpha-1} E_{\mu, \alpha, l}^{\gamma, \delta, k, c}\left(\omega\left(\psi_{2}(x)-\psi_{2}(a)\right)^{\mu} ; p\right) \psi_{2}^{\prime}(t) .
$$

From (14) and given condition of boundedness of $\psi_{1}^{\prime}$, one can have the following integral inequalities:

$$
\begin{aligned}
& \int_{a}^{x}\left(M-\psi_{1}^{\prime}\left(\psi_{2}(t)\right)\right)\left(\psi_{2}(x)-\psi_{2}(t)\right)^{\alpha-1} E_{\mu, \alpha, l}^{\gamma, \delta, k, c}\left(\omega\left(\psi_{2}(x)-\psi_{2}(t)\right)^{\mu} ; p\right) \psi_{2}^{\prime}(t) \mathrm{d} t \\
& \quad \leq\left(\psi_{2}(x)-\psi_{2}(a)\right)^{\alpha-1} E_{\mu, \alpha, l}^{\gamma, \delta, c}\left(\omega\left(\psi_{2}(x)-\psi_{2}(a)\right)^{\mu} ; p\right) \int_{a}^{x}\left(M-\psi_{1}^{\prime}\left(\psi_{2}(t)\right)\right) \psi_{2}^{\prime}(t) \mathrm{d} t, \\
& \int_{a}^{x}\left(M+\psi_{1}^{\prime}\left(\psi_{2}(t)\right)\right)\left(\psi_{2}(x)-\psi_{2}(t)\right)^{\alpha-1} E_{\mu, \alpha, l}^{\gamma, \delta, k, c}\left(\omega\left(\psi_{2}(x)-\psi_{2}(t)\right)^{\mu} ; p\right) \psi_{2}^{\prime}(t) \mathrm{d} t \\
& \quad \leq\left(\psi_{2}(x)-\psi_{2}(a)\right)^{\alpha-1} E_{\mu, \alpha, l}^{\gamma, \delta, k, c} 0\left(\omega\left(\psi_{2}(x)-\psi_{2}(a)\right)^{\mu} ; p\right) \int_{a}^{x}\left(M+\psi_{1}^{\prime}\left(\psi_{2}(t)\right)\right) \psi_{2}^{\prime}(t) \mathrm{d} t .
\end{aligned}
$$


First, we consider inequality (15) as follows:

$$
\begin{aligned}
& M \int_{a}^{x}\left(\psi_{2}(x)-\psi_{2}(t)\right)^{\alpha-1} E_{\mu, \alpha, l}^{\gamma, \delta, k, c}\left(\omega\left(\psi_{2}(x)-\psi_{2}(t)\right)^{\mu} ; p\right) \psi_{2}^{\prime}(t) \mathrm{d} t-\int_{a}^{x}\left(\psi_{2}(x)-\psi_{2}(t)\right)^{\alpha-1} E_{\mu, \alpha, l}^{\gamma, \delta, k, c}\left(\omega\left(\psi_{2}(x)-\psi_{2}(t)\right)^{\mu} ; p\right) \\
& \quad \cdot \psi_{1}^{\prime}\left(\psi_{2}(t)\right) \psi_{2}^{\prime}(t) \mathrm{d} t \\
& \leq\left(\psi_{2}(x)-\psi_{2}(a)\right)^{\alpha-1} E_{\mu, \alpha, l}^{\gamma, \delta, k, c}\left(\omega\left(\psi_{2}(x)-\psi_{2}(a)\right)^{\mu} ; p\right) \int_{a}^{x}\left(M-\psi_{1}^{\prime}\left(\psi_{2}(t)\right)\right) \psi_{2}^{\prime}(t) \mathrm{d} t .
\end{aligned}
$$

Therefore, (17) takes the following form after integrating by parts and using derivative property (11) and a simple computation:

$$
\begin{gathered}
\left(\psi_{2}(x)-\psi_{2}(a)\right)^{\alpha-1} E_{\mu, \alpha, l}^{\gamma, \delta, k, c}\left(\omega\left(\psi_{2}(x)-\psi_{2}(a)\right)^{\mu} ; p\right) \psi_{1}\left(\psi_{2}(x)\right) \\
-\left({ }_{\psi_{2}} \Upsilon_{\mu, \alpha-1, l, \omega, a^{+}}^{\gamma, \delta, k, c} \psi_{1} \circ \psi_{2}\right)(x ; p) \leq M\left(\left(\psi_{2}(x)-\psi_{2}(a)\right)^{\alpha}\right. \\
\left.E_{\mu, \alpha, l}^{\gamma, \delta, k, c}\left(\omega\left(\psi_{2}(x)-\psi_{2}(a)\right)^{\mu} ; p\right)-\left({ }_{\psi_{2}} \Upsilon_{\mu, \alpha-1, l, \omega, a^{+}}^{\gamma, \delta, k, c} 1\right)(x ; p)\right) .
\end{gathered}
$$

Similarly, adopting the same pattern from (16), one can obtain

$$
\begin{gathered}
\left(\psi_{2}(x)-\psi_{2}(a)\right)^{\alpha-1} E_{\mu, \alpha, l}^{\gamma, \delta, k, c}\left(\omega\left(\psi_{2}(x)-\psi_{2}(a)\right)^{\mu} ; p\right) \psi_{1}\left(\psi_{2}(x)\right) \\
-\left({ }_{\psi_{2}} \Upsilon_{\mu, \alpha-1, l, \omega, a^{+}}^{\gamma, \delta, k, c} \psi_{1} \circ \psi_{2}\right)(x ; p) \geq-M\left(\psi_{2}(x)-\psi_{2}(a)\right)^{\alpha} \\
\left.E_{\mu, \alpha, l}^{\gamma, \delta, k, c}\left(\omega\left(\psi_{2}(x)-\psi_{2}(a)\right)^{\mu} ; p\right)-\left({ }_{\psi_{2}} \Upsilon_{\mu, \alpha-1, l, \omega, a^{+}}^{\gamma, \delta, k, c} 1\right)(x ; p)\right) .
\end{gathered}
$$

From (18) and (19), the following inequality is obtained:

$$
\begin{array}{r}
\left|\left(\psi_{2}(x)-\psi_{2}(a)\right)^{\alpha-1} E_{\mu, \alpha, l}^{\gamma, \delta, k, c}\left(\omega\left(\psi_{2}(x)-\psi_{2}(a)\right)^{\mu} ; p\right) \psi_{1}\left(\psi_{2}(x)\right)-\left(\psi_{2} \Upsilon_{\mu, \alpha-1, l, \omega, a^{+}}^{\gamma, \delta, k, c} \psi_{1} \circ \psi_{2}\right)(x ; p)\right| \\
\leq M\left(\left(\psi_{2}(x)-\psi_{2}(a)\right)^{\alpha} E_{\mu, \alpha, l}^{\gamma, \delta, k, c}\left(\omega\left(\psi_{2}(x)-\psi_{2}(a)\right)^{\mu} ; p\right)-\left(\psi_{2} \Upsilon_{\mu, \alpha-1, l, \omega, a^{+}}^{\gamma, \delta, k, c}\right)(x ; p)\right) .
\end{array}
$$

Now, on the contrary, we let $x \in[a, b], t \in[x, b]$, and $\beta \geq 1$. Then, the following inequality holds for Mittag-Leffler function:

$$
\left(\psi_{2}(t)-\psi_{2}(x)\right)^{\beta-1} E_{\mu, \beta, l}^{\gamma, \delta, k, c}\left(\omega\left(\psi_{2}(t)-\psi_{2}(x)\right)^{\mu} ; p\right) \psi_{2}^{\prime}(t) \leq\left(\psi_{2}(b)-\psi_{2}(x)\right)^{\beta-1} E_{\mu, \beta, l}^{\gamma, \delta, k, c}\left(\omega\left(\psi_{2}(b)-\psi_{2}(x)\right)^{\mu} ; p\right) \psi_{2}^{\prime}(t) .
$$

From (21) and the condition of boundedness of $\psi_{1}^{\prime}$, one can have the following integral inequalities:

$$
\begin{aligned}
& \int_{x}^{b}\left(M-\psi_{1}^{\prime}\left(\psi_{2}(t)\right)\right)\left(\psi_{2}(t)-\psi_{2}(x)\right)^{\beta-1} E_{\mu, \beta, l}^{\gamma, \delta, k, c}\left(\omega\left(\psi_{2}(t)-\psi_{2}(x)\right)^{\mu} ; p\right) \psi_{2}^{\prime}(t) \mathrm{d} t \\
& \quad \leq\left(\psi_{2}(b)-\psi_{2}(x)\right)^{\beta-1} E_{\mu, \beta, l}^{\gamma, \delta, k, c}\left(\omega\left(\psi_{2}(b)-\psi_{2}(x)\right)^{\mu} ; p\right) \int_{x}^{b}\left(M-\psi_{1}^{\prime}\left(\psi_{2}(t)\right)\right) \psi_{2}^{\prime}(t) \mathrm{d} t, \\
& \int_{x}^{b}\left(M+\psi_{1}^{\prime}\left(\psi_{2}(t)\right)\right)\left(\psi_{2}(t)-\psi_{2}(x)\right)^{\beta-1} E_{\mu, \beta, l}^{\gamma, \delta, k, c}\left(\omega\left(\psi_{2}(t)-\psi_{2}(x)\right)^{\mu} ; p\right) \psi_{2}^{\prime}(t) \mathrm{d} t \\
& \quad \leq\left(\psi_{2}(b)-\psi_{2}(x)\right)^{\beta-1} E_{\mu, \beta, l}^{\gamma, \delta, k, c}\left(\omega\left(\psi_{2}(b)-\psi_{2}(x)\right)^{\mu} ; p\right) \int_{x}^{b}\left(M+\psi_{1}^{\prime}\left(\psi_{2}(t)\right)\right) \psi_{2}^{\prime}(t) \mathrm{d} t .
\end{aligned}
$$


Following the same procedure as we did for (15) and (16), one can obtain from (22) and (23) the following modulus inequality:

$$
\begin{aligned}
& \left|\left(\psi_{2}(b)-\psi_{2}(x)\right)^{\beta-1} E_{\mu, \beta, l}^{\gamma, \delta, k, c}\left(\omega\left(\psi_{2}(b)-\psi_{2}(x)\right)^{\mu} ; p\right) \psi_{1}\left(\psi_{2}(x)\right)-\left(\psi_{2} \Upsilon_{\mu, \beta-1, l, \omega, b^{-}}^{\gamma, \delta, k, c} \psi_{1} \circ \psi_{2}\right)(x ; p)\right| \\
& \quad \leq M\left(\left(\psi_{2}(b)-\psi_{2}(x)\right)^{\beta} E_{\mu, \beta, l}^{\gamma, \delta, k, c}\left(\omega\left(\psi_{2}(b)-\psi_{2}(x)\right)^{\mu} ; p\right)-\left(\psi_{2} \Upsilon_{\mu, \beta-1, l, \omega, b^{-}}^{\gamma, \delta, k, c}\right)(x ; p)\right) .
\end{aligned}
$$

Inequalities (20) and (24) give (13) which is the required inequality.

In the following, we give direct consequences of above theorem.
Corollary 1. If we put $\alpha=\beta$ in (13), then we get the following fractional integral inequality:

$$
\begin{aligned}
\mid \psi_{1}( & \left.\psi_{2}(x)\right)\left(\psi_{2}(b)-\psi_{2}(x)\right)^{\alpha-1} E_{\mu, \alpha, l}^{\gamma, \delta, k, c}\left(\omega\left(\psi_{2}(b)-\psi_{2}(x)\right)^{\mu} ; p\right)+\left(\psi_{2}(x)-\psi_{2}(a)\right)^{\alpha-1} \times E_{\mu, \alpha, l}^{\gamma, \delta, k, c}\left(\omega\left(\psi_{2}(x)-\psi_{2}(a)\right)^{\mu} ; p\right) \\
& -\left(\left(\psi_{2} \Upsilon_{\mu, \alpha-1, l, \omega, a^{+}}^{\gamma, \delta, k, c} \psi_{1} \circ \psi_{2}\right)(x ; p)+\left(\psi_{2} \Upsilon_{\mu, \alpha-1, l, \omega, b^{-}}^{\gamma, \delta, k, c} \psi_{1} \circ \psi_{2}\right)(x ; p)\right) \mid \\
\leq & M\left(\left(\psi_{2}(x)-\psi_{2}(a)\right)^{\alpha} \times E_{\mu, \alpha, l}^{\gamma, \delta, k, c}\left(\omega\left(\psi_{2}(x)-\psi_{2}(a)\right)^{\mu} ; p\right)+\left(\psi_{2}(b)-\psi_{2}(x)\right)^{\alpha} E_{\mu, \alpha, l}^{\gamma, \delta, k, c}\left(\omega\left(\psi_{2}(b)-\psi_{2}(x)\right)^{\mu} ; p\right)\right. \\
& \left.-\left(\left(\psi_{2} \Upsilon_{\mu, \alpha-1, l, \omega, a^{+}}^{\gamma, \delta, k, c}\right)(x ; p)+\left(\psi_{2} \Upsilon_{\mu, \alpha-1, l, \omega, b^{-}}^{\gamma, \delta, k, c}\right)(x ; p)\right)\right) .
\end{aligned}
$$

\section{Remark 1}

(i) If we put $\psi_{2}(x)=x$ in (13), then we obtain Theorem 5 in [9]

(ii) If we put $\omega=p=0$ and $\psi_{2}(x)=x$ in (13), then we obtain Theorem 1 in [6]

(iii) If we put $\alpha=\beta=1, \psi_{2}(x)=x$, and $\omega=p=0$ in (13), then we obtain Ostrowski inequality (10)

(iv) If we put $\psi_{2}(x)=x$ in (25), then we obtain Corollary 1 in [9]
The next result is a general form of fractional Ostrowski inequality containing generalized Mittag-Leffler function.

Theorem 3. Let $\psi_{1}: I \longrightarrow \mathbb{R}$, where $I$ is an interval in $\mathbb{R}$, be a mapping differentiable in $I^{o}$, the interior of $I$ and $a, b \in I^{\circ}$, $a<b$. If $\psi_{1}$ is integrable function and $m<\psi_{1}^{\prime}\left(\psi_{2}(t)\right) \leq M$ for all $t \in[a, b]$ and $\psi_{2}:[a, b] \longrightarrow \mathbb{R}$ be an increasing and positive function on $(a, b]$, having continuous derivative $\psi_{2}^{\prime}$ on $(a, b)$, then, for $\alpha, \beta \geq 1$, the following inequalities for fractional integrals (6) and (7) hold:

$$
\begin{aligned}
& \psi_{1}\left(\psi_{2}(x)\right)\left(\psi_{2}(x)-\psi_{2}(a)\right)^{\alpha-1} E_{\mu, \alpha, l}^{\gamma, \delta, k, c}\left(\omega\left(\psi_{2}(x)-\psi_{2}(a)\right)^{\mu} ; p\right)-\left(\psi_{2}(b)-\psi_{2}(x)\right)^{\beta-1} \\
& \times E_{\mu, \beta, l}^{\gamma, \delta, k, c}\left(\omega\left(\psi_{2}(b)-\psi_{2}(x)\right)^{\mu} ; p\right)-\left(\psi_{2} \Upsilon_{\mu, \alpha-1, l, \omega, a^{+}}^{\gamma, \delta, k, c} \psi_{1} \circ \psi_{2}\right)(x ; p) \\
&\left.-\left(\psi_{2} \Upsilon_{\mu, \beta-1, l, \omega, b^{-}}^{\gamma, \delta, k, c} \psi_{1} \circ \psi_{2}\right)(x ; p)\right) \leq M\left(\psi_{2}(x)-\psi_{2}(a)\right)^{\alpha} \\
&\left.\times E_{\mu, \alpha, l}^{\gamma, \delta, k, c}\left(\omega\left(\psi_{2}(x)-\psi_{2}(a)\right)^{\mu} ; p\right)-\left(\psi_{2} \Upsilon_{\mu, \alpha-1, l, \omega, a^{+}}^{\gamma, \delta, k, c}\right)(x ; p)\right)-m\left(\left(\psi_{2}(b)-\psi_{2}(x)\right)^{\beta}\right. \\
& \times E_{\mu, \beta, l}^{\gamma, \delta, k, c}\left(\omega\left(\psi_{2}(b)-\psi_{2}(x)\right)^{\mu} ; p\right)-\left(\psi_{2} \Upsilon_{\mu, \beta-1, l, \omega, b^{-}}^{\gamma, \delta, k, c}\right)(x ; p), \\
& \psi_{1}\left(\psi_{2}(x)\right)\left(\psi_{2}(b)-\psi_{2}(x)\right)^{\beta-1} E_{\mu, \beta, l}^{\gamma, \delta, k, c}\left(\omega\left(\psi_{2}(b)-\psi_{2}(x)\right)^{\mu} ; p\right)-\left(\psi_{2}(x)-\psi_{2}(a)\right)^{\alpha-1} \\
& E_{\mu, \alpha, l}^{\gamma, \delta, k, c}\left(\omega\left(\psi_{2}(x)-\psi_{2}(a)\right)^{\mu} ; p\right)-\left(\left(\psi_{2} \Upsilon_{\mu, \alpha-1, l, \omega, a^{+}}^{\gamma, \delta, k, c} \circ \psi_{2}\right)(x ; p)-\left(\psi_{2} \Upsilon_{\mu, \beta-1, l, \omega, b^{-}}^{\gamma, \delta, k, c} \psi_{1} \circ \psi_{2}\right)(x ; p)\right) \\
& \leq \\
& \quad M\left(\left(\psi_{2}(b)-\psi_{2}(x)\right)^{\beta} \times E_{\mu, \beta, l}^{\gamma, \delta, k, c}\left(\omega\left(\psi_{2}(b)-\psi_{2}(x)\right)^{\mu} ; p\right)-\left(\psi_{2} \Upsilon_{\mu, \beta-1, l, \omega, b^{-}}^{\gamma, \delta, k, c}\right)(x ; p)\right) \\
& \quad-m\left(\left(\psi_{2}(x)-\psi_{2}(a)\right)^{\alpha} E_{\mu, \alpha, l}^{\gamma, \delta, k, c}\left(\omega\left(\psi_{2}(x)-\psi_{2}(a)\right)^{\mu} ; p\right)-\left(\psi_{2} \Upsilon_{\mu, \alpha-1, l, \omega, a^{+}}^{\gamma, \delta, k, c} 1\right)(x ; p)\right) .
\end{aligned}
$$


Proof. The proof is similar to the proof of Theorem 2, just after comparing conditions on derivative of $\psi_{1}$, so we left it for the reader.

Some comments on the abovementioned result are given as follows.

\section{Remark 2}

(i) If we put $\omega=p=0$ and $\psi_{2}(x)=x$ in (26) and (27), then we obtain Theorem 1 in [6]

(ii) If we put $m=-M$ in Theorem 3, then with some rearrangements we obtain Theorem 2 (iii) If we put $\psi_{2}(x)=x$ in (26) and (27), then we obtain Theorem 6 in [9]

In the following, we have established a result related to fractional Ostrowski inequality containing generalized Mittag-Leffler function.

Theorem 4. Let $\psi_{1}: I \longrightarrow \mathbb{R}$, where $I$ is an interval in $\mathbb{R}$, be a mapping differentiable in $I^{o}$, the interior of $I$ and $a, b \in I^{o}$, $a<b$. If $\psi_{1}$ is integrable function, $\left|\psi_{1}^{\prime}\left(\psi_{2}(t)\right)\right| \leq M$ for all $t \in[a, b]$ and and $\psi_{2}:[a, b] \longrightarrow \mathbb{R}$ be an increasing and positive function on $(a, b]$, then, for $\alpha, \beta \geq 1$, the following inequality for fractional integrals (6) and (7) holds:

$$
\begin{aligned}
& \mid \psi_{1}\left(\psi_{2}(b)\right)\left(\psi_{2}(b)-\psi_{2}(x)\right)^{\beta-1} E_{\mu, \beta, l}^{\gamma, \delta, k, c}\left(\omega\left(\psi_{2}(b)-\psi_{2}(x)\right)^{\mu} ; p\right)+\psi_{1}\left(\psi_{2}(a)\right) \\
& \cdot\left(\psi_{2}(x)-\psi_{2}(a)\right)^{\alpha-1} E_{\mu, \alpha, l}^{\gamma, \delta, k, c}\left(\omega\left(\psi_{2}(x)-\psi_{2}(a)\right)^{\mu} ; p\right)-\left(\left(\psi_{2} \Upsilon_{\mu, \beta-1, l, \omega, x^{+}}^{\gamma, \delta, k, c} \psi_{1}{ }^{o} \psi_{2}\right)(b ; p)-\left(\psi_{2} \Upsilon_{\mu, \alpha-1, l, \omega, x^{-}}^{\gamma, \delta, k, c} \psi_{1}{ }^{\circ} \psi_{2}\right)(a ; p)\right) \mid \\
& \leq M\left(\left(\psi_{2}(a)-\psi_{2}(x)\right)^{\alpha} \times E_{\mu, \alpha, l}^{\gamma, \delta, k, c}\left(\omega\left(\psi_{2}(x)-\psi_{2}(a)\right)^{\mu} ; p\right)+\left(\psi_{2}(b)-\psi_{2}(x)\right)^{\beta} E_{\mu, \beta, l}^{\gamma, \delta, k, c}\left(\omega\left(\psi_{2}(b)-\psi_{2}(x)\right)^{\mu} ; p\right)\right. \\
& \left.\quad-\left(\left(\psi_{2} \Upsilon_{\mu, \alpha-1, l, \omega, x^{-}}^{\gamma, \delta}\right)(a ; p)+\left(\psi_{2} \Upsilon_{\mu, \beta-1, l, \omega, x^{+}}^{\gamma, \delta, k} 1\right)(b ; p)\right)\right) .
\end{aligned}
$$

Proof. Let $x \in[a, b], t \in[a, x]$, and $\alpha \geq 1$. Then, the following inequality holds true for Mittag-Leffler function:

$$
\begin{aligned}
& \left(\psi_{2}(t)-\psi_{2}(a)\right)^{\alpha-1} E_{\mu, \alpha, l}^{\gamma, \delta, k, c}\left(\omega\left(\psi_{2}(t)-\psi_{2}(a)\right)^{\mu} ; p\right) \psi_{2}^{\prime}(t) \\
& \quad \leq\left(\psi_{2}(x)-\psi_{2}(a)\right)^{\alpha-1} E_{\mu, \alpha, l}^{\gamma, \delta, k, c}\left(\omega\left(\psi_{2}(x)-\psi_{2}(a)\right)^{\mu} ; p\right) \psi_{2}^{\prime}(t) .
\end{aligned}
$$

From (29) and given condition of boundedness on $\psi_{1}^{\prime}$, one can have the following integral inequalities:

$$
\begin{aligned}
& \int_{a}^{x}\left(M-\psi_{1}^{\prime}\left(\psi_{2}(t)\right)\right)\left(\psi_{2}(t)-\psi_{2}(a)\right)^{\alpha-1} E_{\mu, \alpha, l}^{\gamma, \delta, k, c}\left(\omega\left(\psi_{2}(t)-\psi_{2}(a)\right)^{\mu} ; p\right) \psi_{2}^{\prime}(t) \mathrm{d} t \\
& \quad \leq\left(\psi_{2}(x)-\psi_{2}(a)\right)^{\alpha-1} E_{\mu, \alpha, l}^{\gamma, \delta, k, c}\left(\omega\left(\psi_{2}(x)-\psi_{2}(a)\right)^{\mu} ; p\right) \int_{a}^{x}\left(M-\psi_{1}^{\prime}\left(\psi_{2}(t)\right)\right) \psi_{2}^{\prime}(t) \mathrm{d} t, \\
& \int_{a}^{x}\left(M+\psi_{1}^{\prime}\left(\psi_{2}(t)\right)\right)\left(\psi_{2}(t)-\psi_{2}(a)\right)^{\alpha-1} E_{\mu, \alpha, l}^{\gamma, \delta, k, c}\left(\omega\left(\psi_{2}(t)-\psi_{2}(t)\right)^{\mu} ; p\right) \psi_{2}^{\prime}(t) \mathrm{d} t \\
& \quad \leq\left(\psi_{2}(x)-\psi_{2}(a)\right)^{\alpha-1} E_{\mu, \alpha, l}^{\gamma, \delta, k, c}\left(\omega\left(\psi_{2}(x)-\psi_{2}(a)\right)^{\mu} ; p\right) \int_{a}^{x}\left(M+\psi_{1}^{\prime}\left(\psi_{2}(t)\right)\right) \psi_{2}^{\prime}(t) \mathrm{d} t .
\end{aligned}
$$

First, we consider inequality (30) as follows:

$$
\begin{aligned}
& M \int_{a}^{x}\left(\psi_{2}(t)-\psi_{2}(a)\right)^{\alpha-1} E_{\mu, \alpha, l}^{\gamma, \delta, k, c}\left(\omega\left(\psi_{2}(t)-\psi_{2}(a)\right)^{\mu} ; p\right) \psi_{2}^{\prime}(t) \mathrm{d} t \\
& \quad-\int_{a}^{x}\left(\psi_{2}(t)-\psi_{2}(a)\right)^{\alpha-1} E_{\mu, \alpha, l}^{\gamma, \delta, k, c}\left(\omega\left(\psi_{2}(t)-\psi_{2}(a)\right)^{\mu} ; p\right) \psi_{2}^{\prime}(t) \psi_{1}^{\prime}\left(\psi_{2}(t)\right) \mathrm{d} t \\
& \quad \leq\left(\psi_{2}(x)-\psi_{2}(a)\right)^{\alpha-1} E_{\mu, \alpha, l}^{\gamma, \delta, k, c}\left(\omega\left(\psi_{2}(x)-\psi_{2}(a)\right)^{\mu} ; p\right) \int_{a}^{x}\left(M-\psi_{1}^{\prime}\left(\psi_{2}(t)\right)\right) \psi_{2}^{\prime}(t) \mathrm{d} t .
\end{aligned}
$$


Therefore, (32) takes the following form after integrating by parts and using derivative property (11) and a simple computation

$$
\begin{aligned}
& \left(\psi_{2}(x)-\psi_{2}(a)\right)^{\alpha-1} E_{\mu, \alpha, l}^{\gamma, \delta, k, c}\left(\omega\left(\psi_{2}(x)-\psi_{2}(a)\right)^{\mu} ; p\right) \psi_{1}\left(\psi_{2}(a)\right)-\left(\psi_{2} \Upsilon_{\mu, \alpha-1, l, \omega, x^{-}}^{\gamma, \delta, k, c} \psi_{1} \circ \psi_{2}\right)(a ; p) \\
& \quad \leq M\left(\left(\psi_{2}(x)-\psi_{2}(a)\right)^{\alpha} \times E_{\mu, \alpha, l}^{\gamma, \delta, k, c}\left(\omega\left(\psi_{2}(x)-\psi_{2}(a)\right)^{\mu} ; p\right)-\left(\psi_{2} \Upsilon_{\mu, \alpha-1, l, \omega, x^{-}}^{\gamma, \delta, k, c}\right)(a ; p)\right) .
\end{aligned}
$$
obtain

Similarly, adopting the same pattern from (31), one can

$$
\begin{gathered}
\left(\psi_{2}(x)-\psi_{2}(a)\right)^{\alpha-1} E_{\mu, \alpha, l}^{\gamma, \delta, k, c}\left(\omega\left(\psi_{2}(x)-\psi_{2}(a)\right)^{\mu} ; p\right) \psi_{1}\left(\psi_{2}(a)\right)-\left(\psi_{2} \Upsilon_{\mu, \alpha-1, l, \omega, x^{-}}^{\gamma, \delta, k, c} \psi_{1} \circ \psi_{2}\right)(a ; p) \\
\geq-M\left(\left(\psi_{2}(x)-\psi_{2}(a)\right)^{\alpha} \times E_{\mu, \alpha, l}^{\gamma, \delta, k, c}\left(\omega\left(\psi_{2}(x)-\psi_{2}(a)\right)^{\mu} ; p\right)-\left(\psi_{2} \Upsilon_{\mu, \alpha-1, l, \omega, x^{-}}^{\gamma, \delta, k, c} 1\right)(a ; p)\right) .
\end{gathered}
$$

From (33) and (34), the following inequality is obtained:

$$
\begin{aligned}
& \left|\left(\psi_{2}(x)-\psi_{2}(a)\right)^{\alpha-1} E_{\mu, \alpha, l}^{\gamma, \delta, k, c}\left(\omega\left(\psi_{2}(x)-\psi_{2}(a)\right)^{\mu} ; p\right) \psi_{1}\left(\psi_{2}(a)\right)-\left(\psi_{2} \Upsilon_{\mu, \alpha-1, l, \omega, x^{-}}^{\gamma, \delta, k, c} \psi_{1} \circ \psi_{2}\right)(a ; p)\right| \\
& \quad \leq M\left(\left(\psi_{2}(x)-\psi_{2}(a)\right)^{\alpha} \times E_{\mu, \alpha, l}^{\gamma, \delta, k, c}\left(\omega\left(\left(\psi_{2}(x)-\psi_{2}(a)\right)^{\alpha}\right)^{\mu} ; p\right)-\left(\psi_{2} \Upsilon_{\mu, \alpha-1, l, \omega, x^{-}}^{\gamma, \delta, k}\right)(a ; p)\right) .
\end{aligned}
$$

Now, on the contrary, we let $x \in[a, b], t \in[x, b]$, and $\beta \geq 1$. Then, the following inequality holds for Mittag-Leffler function:

$$
\begin{aligned}
& \left(\psi_{2}(b)-\psi_{2}(t)\right)^{\beta-1} E_{\mu, \beta, l}^{\gamma, \delta, k, c}\left(\omega\left(\psi_{2}(b)-\psi_{2}(t)\right)^{\mu} ; p\right) \psi_{2}^{\prime}(t) \leq\left(\psi_{2}(b)-\psi_{2}(x)\right)^{\beta-1} \\
& \quad \times E_{\mu, \beta, l}^{\gamma, \delta, k, c}\left(\omega\left(\psi_{2}(b)-\psi_{2}(x)\right)^{\mu} ; p\right) \psi_{2}^{\prime}(t) .
\end{aligned}
$$

From (36) and given condition of boundedness of $\psi_{1}^{\prime}$, one can have the following integral inequalities:

$$
\begin{aligned}
& \int_{x}^{b}\left(M-\psi_{1}^{\prime}\left(\psi_{2}(t)\right)\right)\left(\psi_{2}(b)-\psi_{2}(t)\right)^{\beta-1} E_{\mu, \beta, l}^{\gamma, \delta, k, c}\left(\omega\left(\psi_{2}(b)-\psi_{2}(t)\right)^{\mu} ; p\right) \psi_{2}^{\prime}(t) \mathrm{d} t \\
& \quad \leq\left(\psi_{2}(b)-\psi_{2}(x)\right)^{\beta-1} E_{\mu, \beta, l}^{\gamma, \delta, k, c}\left(\omega\left(\psi_{2}(b)-\psi_{2}(x)\right)^{\mu} ; p\right) \int_{x}^{b}\left(M-\psi_{1}^{\prime}\left(\psi_{2}(t)\right)\right) \psi_{2}^{\prime}(t) \mathrm{d} t, \\
& \int_{x}^{b}\left(M+\psi_{1}^{\prime}\left(\psi_{2}(t)\right)\right)\left(\psi_{2}(b)-\psi_{2}(t)\right)^{\beta-1} E_{\mu, \beta, l}^{\gamma, \delta, k, c}\left(\omega\left(\psi_{2}(b)-\psi_{2}(t)\right)^{\mu} ; p\right) \psi_{2}^{\prime}(t) \mathrm{d} t \\
& \quad \leq\left(\psi_{2}(b)-\psi_{2}(x)\right)^{\beta-1} E_{\mu, \beta, l}^{\gamma, \delta, k, c}\left(\omega\left(\psi_{2}(b)-\psi_{2}(x)\right)^{\mu} ; p\right) \int_{x}^{b}\left(M+\psi_{1}^{\prime}\left(\psi_{2}(t)\right)\right) \psi_{2}^{\prime}(t) \mathrm{d} t .
\end{aligned}
$$


Following the same procedure as we did for (30) and (31), one can obtain from (37) and (38) the following modulus inequality:

$$
\begin{aligned}
\mid\left(\psi_{2}(b)-\right. & \left.\psi_{2}(x)\right)^{\beta-1} E_{\mu, \beta, l}^{\gamma, \delta, k, c}\left(\omega\left(\psi_{2}(b)-\psi_{2}(x)\right)^{\mu} ; p\right) \psi_{1}\left(\psi_{2}(b)\right)-\left(\psi_{2} \Upsilon_{\mu, \beta-1, l, \omega, x^{+}}^{\gamma, \delta, k, c} \psi_{1} \circ \psi_{2}\right)(b ; p) \mid \\
\leq & M\left(\left(\psi_{2}(b)-\psi_{2}(x)\right)^{\beta} \times E_{\mu, \beta, l}^{\gamma, \delta, k, c}\left(\omega\left(\psi_{2}(b)-\psi_{2}(x)\right)^{\mu} ; p\right)-\left(\psi_{2} \Upsilon_{\mu, \beta-1, l, \omega, x^{+}}^{\gamma, \delta, k, c}\right)(b ; p)\right) .
\end{aligned}
$$

Inequalities (35) and (39) give (28) which is required inequality.

Some direct consequences of the above theorem are given below.
Corollary 2. If we put $\alpha=\beta$ in (28), then we get the following fractional integral inequality:

$$
\begin{aligned}
& \mid \psi_{1}\left(\psi_{2}(b)\right)\left(\psi_{2}(b)-\psi_{2}(x)\right)^{\alpha-1} E_{\mu,,, l}^{\gamma, \delta, k, c}\left(\omega\left(\psi_{2}(b)-\psi_{2}(x)\right)^{\mu} ; p\right)+\psi_{1}\left(\psi_{2}(a)\right) \\
& \quad \cdot\left(\psi_{2}(x)-\psi_{2}(a)\right)^{\alpha-1} E_{\mu, \alpha, l}^{\gamma, \delta, k, c}\left(\omega\left(\psi_{2}(x)-\psi_{2}(a)\right)^{\mu} ; p\right)-\left(\left(\psi_{2} Y_{\mu, \alpha-1, l, \omega, x^{+}}^{\gamma, \delta, k} \psi_{1} \circ \psi_{2}\right)(b ; p)+\left(\psi_{2} \Upsilon_{\mu, \alpha-1, l, \omega, x^{-}}^{\gamma, \delta, k, c} \psi_{1} \circ \psi_{2}\right)(a ; p)\right) \mid \\
& \quad \leq M\left(\left(\psi_{2}(x)-\psi_{2}(a)\right)^{\alpha} \times E_{\mu, \alpha, l}^{\gamma, \delta, k, c}\left(\omega\left(\psi_{2}(x)-\psi_{2}(a)\right)^{\mu} ; p\right)+\left(\psi_{2}(b)-\psi_{2}(x)\right)^{\alpha} E_{\mu, \alpha, l}^{\gamma, \delta, k, c}\left(\omega\left(\psi_{2}(b)-\psi_{2}(x)\right)^{\mu} ; p\right)\right. \\
& \left.\quad-\left(\left(\psi_{2} Y_{\mu, \alpha-1, l, \omega, x^{-}}^{\gamma, \delta, k}\right)(a ; p)+\left(\psi_{2} Y_{\mu, \alpha-1, l, \omega, x^{+}}^{\gamma, \delta, k, c} 1\right)(b ; p)\right)\right) .
\end{aligned}
$$

\section{Remark 3}

(i) If we put $\psi_{2}(x)=x$ in (28), then we obtain Theorem 5 in [9]

(ii) If we put $\omega=p=0$ and $\psi_{2}(x)=x$ in (28), then we obtain Theorem 1 in [6]

(iii) If we put $\psi_{2}(x)=x$ in (40), then we obtain Corollary 2 in [9]

\section{Applications}

In this section, we just describe some applications of Theorem 4 and leave such applications of other results for the reader. By applying Theorem 4 at end points of the interval $[a, b]$ and adding the resulting inequalities, one obtains the error bounds of compact form of the fractional Hadamard inequality.

Theorem 5. Under the assumptions of Theorem 4, the following estimation of Hadamard inequality can be obtained:

$$
\begin{aligned}
& \mid \frac{\left(\psi_{1}\left(\psi_{2}(b)\right)+\psi_{1}\left(\psi_{2}(b)\right)\right)}{2}\left(\psi_{2} \Upsilon_{\mu, \alpha-1, l, \omega, a^{+}}^{\gamma, \delta, k, c} 1\right)(b ; p)-\frac{1}{2}\left(\left(\psi_{2} \Upsilon_{\mu, \alpha-1, l, \omega, b^{-}}^{\gamma, \delta, k, c} \psi_{1} \circ \psi_{2}\right)(a ; p)+\left(\psi_{2} \Upsilon_{\mu, \alpha-1, l, \omega, a^{+}}^{\gamma, \delta, k, c} \psi_{1} \circ \psi_{2}\right)(b ; p) \mid\right. \\
& \leq \leq \frac{M}{2}\left(\psi_{2}(b)-\psi_{2}(a)^{\alpha}\right) E_{\mu, \alpha, l}^{\gamma, \delta, k, c}\left(\omega\left(\psi_{2}(b)-\psi_{2}(a)\right)^{\mu} ; p\right)-\frac{M}{2}\left(\left(\psi_{2} \Upsilon_{\mu, \alpha-1, l, \omega, b^{-}}^{\gamma, \delta, k, c} 1\right)(a ; p)\right. \\
& \left.\left.\quad+\left(\psi_{2} \Upsilon_{\mu, \alpha-1, l, \omega, a^{+}}^{\gamma, \delta, k, c} 1\right)(b ; p)\right)\right) .
\end{aligned}
$$

Proof. By putting $x=a, \alpha=\beta$, and $x=b$ in (40) then adding the resulting inequalities, we obtain

$$
\begin{aligned}
& \mid\left(\psi_{1}\left(\psi_{2}(b)\right)+\psi_{1}\left(\psi_{2}(b)\right)\right)\left(\psi_{2}(b)-\psi_{2}(a)\right)(a)^{\alpha-1} E_{\mu, \alpha, l}^{\gamma, \delta, k, c}\left(\omega\left(\psi_{2}(b)-\psi_{2}(a)\right)^{\mu} ; p\right) \\
& \quad-\left(\left(\psi_{2} \Upsilon_{\mu, \alpha-1, l, \omega, b^{-}}^{\gamma, \delta, k, c} \psi_{1} \circ \psi_{2}\right)(a ; p)+\left(\psi_{2} \Upsilon_{\mu, \alpha-1, l, \omega, a^{+}}^{\gamma, \delta, k, c} \psi_{1} \circ \psi_{2}\right)(b ; p)\right) \mid \\
& \left.\quad \leq M\left(\psi_{2}(b)-\psi_{2}(a)\right)^{\alpha} E_{\mu, \alpha, l}^{\gamma, \delta, k, c}\left(\omega\left(\psi_{2}(b)-\psi_{2}(a)\right)^{\mu} ; p\right)-\left(\left(\psi_{2} \Upsilon_{\mu, \alpha-1, l, l, \omega, b^{-}}^{\gamma, \delta, c, c}\right)(a ; p)+\left(\psi_{2} \Upsilon_{\mu, \alpha-1, l, \omega, a^{+}}^{\gamma, \delta, k, c} 1\right)(b ; p)\right)\right) .
\end{aligned}
$$


Multiplying both sides of the above inequality by $1 / 2$ and using (8) and (9), inequality (41) can be obtained.
Theorem 6. Under the assumptions of Theorem 4, the following inequality can be obtained:

Remark 4. If in (41) $\alpha$ is replaced by $\alpha+1$ and $\omega$ by $\omega \prime=\omega /\left((g(b)-g(a))^{\mu}\right)$, then we get an error bound of the Hadamard inequality given in Theorem 1 in [20].

$$
\begin{aligned}
& \mid \psi_{1}\left(\psi_{2}(b)\right)\left(\psi_{2} \Upsilon_{\mu, \alpha-1, l, \omega, b^{-}}^{\gamma, \delta, k, c} 1\right)\left(\frac{a+b}{2} ; p\right)+\psi_{1}\left(\psi_{2}(a)\right)\left(\psi_{2} \Upsilon_{\mu, \alpha-1, l, \omega, a^{+}}^{\gamma, \delta, k, c} 1\right)\left(\frac{a+b}{2} ; p\right) \\
&-\left(\left(\psi_{2} \Upsilon_{\mu, \alpha-1, l, \omega,(a+b / 2)^{+}}^{\gamma, \delta, k, c} \psi_{1} \circ \psi_{2}\right)(b ; p)+\left(\psi_{2} \Upsilon_{\mu, \alpha-1, l, \omega,(a+b / 2)^{-}}^{\gamma, \delta, k, c} \psi_{1} \circ \psi_{2}\right)(a ; p)\right) \mid \\
& \leq M\left(\left(\psi_{2}\left(\frac{a+b}{2}\right)-\psi_{2}(a)\right)^{\alpha} E_{\mu, \alpha, l}^{\gamma, \delta, k, c}\left(\omega\left(\psi_{2}\left(\frac{a+b}{2}\right)-\psi_{2}(a)\right)^{\mu} ; p\right)\right. \\
&+\left(\psi_{2}(b)-\psi_{2}\left(\frac{a+b}{2}\right)\right)^{\alpha} E_{\mu, \alpha, l}^{\gamma, \delta, k, c}\left(\omega\left(\psi_{2}(b)-\psi_{2}\left(\frac{a+b}{2}\right)\right)^{\mu} ; p\right) \\
&\left.-\left(\left(\psi_{2} \Upsilon_{\mu, \alpha-1, l, \omega,(a+b / 2)^{-}}^{\gamma, \delta, k, c}\right)(a ; p)+\left(\psi_{2} \Upsilon_{\mu, \alpha-1, l, \omega,(a+b / 2)^{+}}^{\gamma, \delta, k, c}\right)(b ; p)\right)\right) .
\end{aligned}
$$

Proof. By putting $x=(a+b) / 2$ for $\alpha=\beta$ in (40), (43) can be obtained.

\section{Concluding Remarks}

We have established generalized fractional integral inequalities of Ostrowski type. By applying boundedness of a differentiable function and using properties of an extended generalized Mittag-Leffler function different generalized versions of Ostrowski type inequalities are analyzed. Also, some deductions from results of this paper are connected with already published results. Furthermore, all the results can be calculated for fractional integral operators defined in $[2,3,5,6,27]$, and we left it for the reader.

\section{Data Availability}

No data were used to support the study.

\section{Conflicts of Interest}

The authors do not have any conflicts of interest.

\section{Authors' Contributions}

All authors have equal contributions.

\section{Acknowledgments}

This research work was supported by the Higher Education Commission of Pakistan under NRPU 2016, Project no. 5421.

\section{References}

[1] A. Wiman, "Über den fundamentalsatz in der teorie der funktionen ea(x)," Acta Mathematica, vol. 29, pp. 191-201, 1905.

[2] T. R. Prabhakar, "A singular integral equation with a generalized Mittag-Leffler function in the kernel," Yokohama Mathematical Journal, vol. 19, pp. 7-15, 1971.

[3] A. K. Shukla and J. C. Prajapati, "On a generalization of Mittag-Leffler function and its properties," Journal of Mathematical Analysis and Applications, vol. 336, no. 2, pp. 797-811, 2007.

[4] T. O. Salim, "Some properties relating to the generalized Mittag-Leffler function," Advances in Applied Mathematics and Mechanics, vol. 4, pp. 21-30, 2009.

[5] T. O. Salim and A. W. Faraj, "A Generalization of MittafLeffler function and integral operator associated with integral calculus," Journal Fraction Calculator Application, vol. 3, no. 5, pp. 1-13, 2012.

[6] G. Rahman, D. Baleanu, M. Al Qurashi, S. D. Purohit, S. Mubeen, and M. Arshad, "The extended Mittag-Leffler function via fractional calculus," The Journal of Nonlinear Sciences and Applications, vol. 10, no. 8, pp. 4244-4253, 2017.

[7] M. Andrić, G. Farid, and J. Pečarić, "A further extension of Mittag-Leffler function," Fractional Calculus and Applied Analysis, vol. 21, no. 4, pp. 1377-1395, 2018.

[8] G. Farid, "Some new Ostrowski type inequalities via fractional integrals," The International Journal of Nonlinear Analysis and Applications, vol. 14, no. 1, pp. 64-68, 2017.

[9] G. Farid, U. N. Katugampola, and M. Usman, "Ostrowskitype fractional integral inequalities for mappings whose derivatives are h-convex via Katugampola fractional integrals," Studia Universitatis Babes-Bolyai Matematica, vol. 63, no. 4, pp. 465-474, 2018.

[10] G. Farid, S. Mubeen, and E. Set, "Fractional inequalities associated with a generalized mittag-leffler function and 
applications," International Journal of Analysis and Applications, vol. 17, no. 4, 2019.

[11] Y. C. Kwun, G. Farid, W. Nazeer, S. Ullah, and S. M. Kang, "Generalized riemann-liouville \$ $\$$-fractional integrals associated with Ostrowski type inequalities and error bounds of hadamard inequalities," IEEE Access, vol. 6, pp. 64946-64953, 2018.

[12] M. A. Khan, T. Ali, S. S. Dragomir, and M. Z. Sarikaya, "Hermite-Hadamard Type Inequalities for conformable fractional integrals," Revista de la Real Academia de Ciencias Exactas, Físicas y Naturales. Serie A. Matemáticas, vol. 112, no. 4, pp. 1033-1048, 2018.

[13] M. A. Khan, S. Begum, Y. Khurshid, and Y. M. Chu, "Ostrowski Type Inequalities involving conformable fractional integrals," Journal of Inequalities and Applications, vol. 70, pp. 1-14, 2018.

[14] M. A. Khan, M. Iqbal, M. SulEman, and Y. M. Chu, "HermiteHadamard Type Inequalities for fractional integrals via green functions," Journal of Inequalities and Applications, vol. 161, pp. 1-15, 2018.

[15] M. A. Khan, Y. M. Chu, A. Kashuri, R. Liko, and G. Ali, "Conformable fractional integrals versions of HermiteHadamard inequalities and generalizations," Journal of Function Spaces, vol. 2018, Article ID 6928130, 9 pages, 2018.

[16] Y. Rao, M. Yussouf, G. Farid, J. Pečarić, and I. Tlili, "Further generalizations of Hadamard and Feéjer Hadamard fractional inequalities and error estimations," Advances in Difference Equations.

[17] G. Farid, "A unified integral operator and further its consequences," Open Journal of Mathematical Analysis, vol. 4, no. 1, pp. 1-7, 2020.

[18] Y. C. Kwun, G. Farid, S. Ullah, W. Nazeer, K. Mahreen, and S. M. Kang, "Inequalities for a unified integral operator and associated results in fractional calculus," IEEE Access, vol. 7, pp. 126283-126292, 2019.

[19] A. Ostrowski, "Über die Absolutabweichung einer dierentierbaren Funktion von ihren Integralmittelwert," Commentarii Mathematici Helvetici, vol. 10, pp. 226-227, 1938.

[20] S. S. Dragomir and T. M. Rassias, Ostrowski-type Inequalities and Applications in Numerical Integration, Kluwer Academic Publishers, Dordrecht, Boston, London, 2002.

[21] S. S. Dragomir and S. Wang, "An inequality of OstrowskiGrüss' type and its applications to the estimation of error bounds for some special means and for some numerical quadrature rules," Computers \& Mathematics with Applications, vol. 33, no. 11, pp. 15-20, 1997.

[22] G. Farid, "New ostrowski-type inequalities and their applications in two coordinates," Acta Mathematica Universitatis Comenianae, vol. 85, no. 1, 2016.

[23] G. Farid, "Straightforward proofs of Ostrowski inequality and some related results," International Journal of Analysis, vol. 2016, Article ID 3918483, 5 pages, 2016.

[24] G. Farid, S. Rafique, and A. U. Rehman, "More on Ostrowski and ostrowski-gruss type inequalities," Communications in Optimization Theory, vol. 2017, p. 9, 2017.

[25] G. Farid and A. U. Rehman, "On Chebyshev functional and Ostrowski-Gruss type inequalities for two coordinates," International Journal of Analysis and Applications, vol. 12, no. 2, pp. 180-187, 2016.

[26] X. Qiaoling, Z. Jian, and L. Wenjun, "A new generalization of Ostrowski-type inequality involving functions of two independent variables," Computers \& Mathematics with Applications, vol. 60, pp. 2219-2224, 2010.
[27] H. M. Srivastava and Ž. Tomovski, "Fractional calculus with an integral operator containing a generalized Mittag-Leffler function in the kernel," Applied Mathematics and Computation, vol. 211, no. 1, pp. 198-210, 2009. 